\title{
Optimizing glucose control for diabetic patients undergoing percutaneous coronary intervention
}

\author{
Haseong Chang and Sung-Hea Kim
}

Division of Cardiology, Department of Internal Medicine, Konkuk University Medical Center, Konkuk University School of Medicine, Seoul, Korea
Received: October 2, 2021 Accepted: October 18, 2021

\section{Correspondence to} Sung-Hea Kim, M.D.

Division of Cardiology, Department of Internal Medicine, Konkuk University Medical Center, Konkuk University School of Medicine, 120-1 Neungdong-ro, Gwangjin-gu, Seoul 05030, Korea Tel: +82-2-2030-6061

Fax: $+82-2-2030-6063$

E-mail: shkim@kuh.ac.kr https://orcid.org/0000-00015809-7958

\section{See Article on Page 1365-1376}

Diabetes mellitus (DM) is a major risk factor for atherosclerotic cardiovascular disease. Lowering blood glucose is an important medical intervention to prevent recurrence in patients with established atherosclerotic cardiovascular disease. However, lower has not been always better, until now.

Intensive glucose lowering improved the microvascular complications of DM, but not the macrovascular complications [1], with increased risks of hypoglycemia and mortality [2]. The paradoxical increase in mortality with aggressive glucose lowering, a U-shaped curve, has been reported in the general DM population $[3,4]$. Hypoglycemia during aggressive glucose lowering therapy has adverse effects on the cardiovascular system and worsens clinical outcomes [5].

There are several proposed explanations for worse outcomes with aggressive glucose lowering in DM patients. Patients experiencing hypoglycemic events generally have a poor nutritional state. Hypoglycemia causes oxidative stress and vascular inflammation. In addition, hypoglycemia activates the adrenergic system, and a hyperadrenergic state increases arrhythmogenic potential.

The relationship between hemoglobin Arc (HbAic) and clinical outcome after percutaneous coronary intervention
(PCI) is still debated. Hwang et al. [6] reported that a lower HbAic level 2 years after PCI was associated with a decreased risk of major adverse cardiovascular events, mostly driven by a lower rate of target lesion revascularization. Corpus et al. [7] showed an association between a lower HbAic level at the time of PCI and a decreased rate of target vessel revascularization, but another study by Lemesle et al. [8] failed to show a benefit of a lower HbArc level.

The association between aggressive glucose lowering and an increased risk of mortality is not as evident in PCI patients as in the general DM population. Two recent studies reported worse outcomes after intensive glucose lowering in PCI patients. A Japanese study showed that tight glycemic control was associated with a higher risk of cardiovascular death compared with mild glycemic control in diabetics who underwent PCI [9]. A recently published single-center retrospective study in diabetics undergoing PCI showed that a low HbAic level was associated with an increased risk of mortality, whereas a higher level was associated with an increased risk of myocardial infarction (MI) [10].

In this issue of the Korean Journal of Internal Medicine, Bae et al. [11] showed a lower incidence of major adverse cardiac and cerebrovascular events in patients with aggressive glycemic control. 
The benefit of a lower HbıAc was driven mainly by a lower rate of stroke. Poor glycemic control induces prothrombotic states and causes thrombotic adverse events such as MI and revascularization. An increased risk of stroke in the uncontrolled group in this study is consistent with previous studies that showed an increased risk of thrombotic events in poorly controlled DM.

Compared with previous studies, this study has several strengths. The authors used the mean $\mathrm{HbAic}$ level during follow-up instead of the initial $\mathrm{HbArc}$ level at the time of PCI. Previous studies used the HbArc level at a single time point such as at the time of PCI or 2 years after PCI. In this study, the average HbAlc level during follow-up reflected better the true levels of blood glucose during the study period compared with other studies. Another strength is the long-term follow-up duration (median duration $>6$ years).

This study also had limitations, inherent in the retrospective study design. The groups were divided according to the achieved HbA1c level, and not by actual intensity of the glucose lowering treatment. Thus, the low $\mathrm{HbAlc} \mathrm{level} \mathrm{in} \mathrm{the} \mathrm{active} \mathrm{control} \mathrm{(AC)} \mathrm{group} \mathrm{in} \mathrm{this} \mathrm{study}$ was not necessarily the result of aggressive diabetes control but may also be because cases of easily controlled diabetes were included. The duration of DM was shorter in the AC group, and fewer patients in this group were on either an oral hypoglycemic agent or insulin treatment at baseline. Thus, there is the possibility that patients in the AC group may have had a lower atherosclerotic burden; therefore, a further randomized study is warranted to address these limitations.

The advent of antidiabetic medications with proven cardiovascular benefits is changing the DM treatment paradigm in the PCI population, from a focus on the blood glucose level to use of drug combinations tailored to improve cardiovascular outcomes. The DM treatment guidelines recommend the use of specific drugs with proven cardiovascular benefits for established cardiovascular disease, independent of the baseline HbArc level [12].

Nonetheless, the challenge against the U-shaped curve will not cease in the era of new DM agents with less hypoglycemic side effects and proven cardiovascular benefits.

\section{Conflict of interest}

No potential conflict of interest relevant to this article was reported.

\section{REFERENCES}

1. ADVANCE Collaborative Group, Patel A, MacMahon S, et al. Intensive blood glucose control and vascular outcomes in patients with type 2 diabetes. $\mathrm{N}$ Engl J Med 2008;358:2560-2572.

2. Action to Control Cardiovascular Risk in Diabetes Study Group, Gerstein HC, Miller ME, et al. Effects of intensive glucose lowering in type 2 diabetes. N Engl J Med 2008;358:2545-2559.

3. Currie CJ, Peters JR, Tynan A, et al. Survival as a function of $\mathrm{HbA}(1 \mathrm{c})$ in people with type 2 diabetes: a retrospective cohort study. Lancet 2010;375:481-489.

4. Huang ES, Liu JY, Moffet HH, John PM, Karter AJ. Glycemic control, complications, and death in older diabetic patients: the diabetes and aging study. Diabetes Care 2011;34:1329-1336.

5. Connelly KA, Yan AT, Leiter LA, Bhatt DL, Verma S. Cardiovascular implications of hypoglycemia in diabetes mellitus. Circulation 2015;132:2345-2350.

6. Hwang JK, Lee SH, Song YB, et al. Glycemic control status after percutaneous coronary intervention and longterm clinical outcomes in patients with type 2 diabetes mellitus. Circ Cardiovasc Interv 2017;10:eoo4157.

7. Corpus RA, George PB, House JA, et al. Optimal glycemic control is associated with a lower rate of target vessel revascularization in treated type II diabetic patients undergoing elective percutaneous coronary intervention. J Am Coll Cardiol 2004;43:8-14.

8. Lemesle G, Bonello L, de Labriolle A, et al. Prognostic value of hemoglobin $\mathrm{A}_{1} \mathrm{C}$ levels in patients with diabetes mellitus undergoing percutaneous coronary intervention with stent implantation. Am J Cardiol 2009;104:41-45.

9. Funamizu T, Iwata H, Nishida $Y$, et al. Increased risk of cardiovascular mortality by strict glycemic control (pre-procedural HbA1c $<6.5 \%$ ) in Japanese medically-treated diabetic patients following percutaneous coronary intervention: a 10-year follow-up study. Cardiovasc Diabetol 2020;19:21.

10. Baber U, Azzalini L, Masoomi R, et al. Hemoglobin Aic 
and cardiovascular outcomes following percutaneous coronary intervention: insights from a large single-center registry. JACC Cardiovasc Interv 2021;14:388-397.

11. Bae J, Yoon JH, Lee JH, et al. Long-term effects of the mean hemoglobin Aic levels after percutaneous coronary intervention in patients with diabetes. Korean J Intern
Med 2021;36:1365-1376.

12. American Diabetes Association. 9. Pharmacologic approaches to glycemic treatment: standards of medical care in diabetes-2021. Diabetes Care 2021;44(Suppl 1):S111-S124. 